\title{
Immunogenicity Specimen Assessments Result Standard Unit
}

National Cancer Institute

\section{Source}

National Cancer Institute. Immunogenicity Specimen Assessments Result Standard Unit. NCI Thesaurus. Code C117563.

The standard unit of measure for the immunogenicity specimen assessment results. 